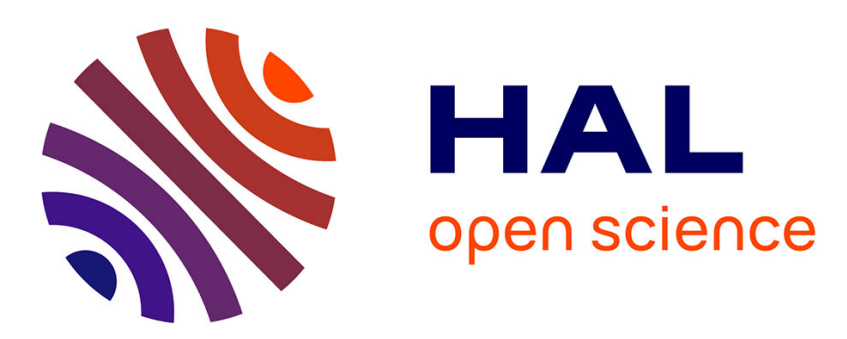

\title{
On the interval estimation for nonlinear singular system
}

Gang Zheng, Denis Efimov, Francisco Javier Bejarano, Wilfrid Perruquetti

\section{To cite this version:}

Gang Zheng, Denis Efimov, Francisco Javier Bejarano, Wilfrid Perruquetti. On the interval estimation for nonlinear singular system. 54th IEEE Conference on Decision and Control (CDC), Dec 2015, Osaka, Japan. hal-01252374

\section{HAL Id: hal-01252374 \\ https://hal.inria.fr/hal-01252374}

Submitted on 7 Jan 2016

HAL is a multi-disciplinary open access archive for the deposit and dissemination of scientific research documents, whether they are published or not. The documents may come from teaching and research institutions in France or abroad, or from public or private research centers.
L'archive ouverte pluridisciplinaire HAL, est destinée au dépôt et à la diffusion de documents scientifiques de niveau recherche, publiés ou non, émanant des établissements d'enseignement et de recherche français ou étrangers, des laboratoires publics ou privés. 


\title{
On the interval estimation for nonlinear singular system
}

\author{
G. Zheng, D, Efimov, F. J. Bejarano and W. Perruquetti
}

\begin{abstract}
This paper investigates the interval observer design problem for a class of nonlinear singular systems with uncertainties in the state and in the output. Although the asymptotic estimation of the state for this class of systems may be not possible, it has been shown that, under suitable assumptions, an interval observer can be designed to provide the upper and the lower estimations of the real state. Moreover, this technique can be applied to unobservable nonlinear singular systems to obtain the interval estimation. The proposed result can be easily extended to deal with the nonlinear singular systems with parameter uncertainty as well.
\end{abstract}

\section{INTRODUCTION}

Singular system approach was introduced to model a large class of systems [8], [7]. In [22], the authors have studied the solvability, controllability and observability concepts for singular systems with regular matrix pencil. The algebraic duality between controllability and observability for singular systems with regular matrix pencil is proven by using the Schwartz distribution framework in [9]. In [15], the concept of casual observability was proposed for singular systems. The strong observability and strong detectability of a general class of singular linear systems with unknown inputs are recently tackled in [3], [1] by converting the singular system into a regular one with unknown inputs and algebraic constraints. The observability problem for nonlinear singular system has been treated in [4], [2].

Concerning the observer design, a Luenberger-like observer has been proposed in [18] for linear singular systems. [11] gave necessary and sufficient condition for the existence of a reduced order observer for the linear singular systems with known inputs, and the result was extended to treat the linear singular systems with unknown inputs in [12]. In [16], a generalized observer was studied by involving the derivative of input and output. For the nonlinear singular system, [17] studied an observer for a class of nonlinear singular systems in which the system was linearized around the equilibrium point. The same technique was used in [6] to study a reduced order observer for a class of nonlinear singular systems. And recently, the technique of regularization by applying the geometrical differential method to the nonlinear singular systems was introduced in [5].

Gang Zheng, D, Efimov and Wilfrid Perruquetti are with Project Non-A, INRIA Lille-Nord Europe, 40 avenue Halley and CRIStAL UMR CNRS 9189, Ecole Centrale de Lille, 59650 Villeneuve d'Ascq, France. Francisco Javier Bejarano is with SEPI, ESIME Ticomn, IPN, Av. San Jos Ticomn 600, C.P. 07340, Mexico City, Mexico. This paper was supported by Ministry of Higher Education and Research, Nord-Pas de Calais Regional Council and FEDER through the Contrat de Projets Etat Region (CPER) CIA 20072013, and also supported by ARCIR Project ESTIREZ, Nord-Pas de Calais Regional Council, France. F.J. Bejarnano acknowledges as well the support of Proyecto SIP 20151040, Mexico.
Most of the cited references are for the asymptotic estimation of the state for singular systems without uncertainties. The observer design becomes complicated when considering the systems with uncertain terms in the state and in the measurement. In this situation, the exact estimation may be not possible, and one solution is to provide the upper and lower bound estimation of the admissible values for the state by applying the theory of set-membership or interval estimation [14], [21], [20], [19]

Concerning the interval observer design for singular system, the only work existing in the literature is [13], where the problem of interval observer design is addressed for a class of linear singular systems with delays. In this work, the studied singular system was decoupled into two parts $x=\left(x_{1}, x_{2}\right)^{T}$ : the dynamical one and the algebraic one. The authors then proposed an interval observer by restrictively assuming that $x_{2}$ can be fully described as the function of $x_{1}$. Thus the algebraic equation can be removed and the studied singular system is equivalent to a regular one. Without imposing this restrictive condition, this paper proposes a method to design an interval observer for a class of nonlinear singular systems with uncertainties. By imposing the observability rank condition for the linear part and assuming some boundedness properties of the studied system, this paper shows that an interval observer for this class of uncertain nonlinear singular systems always exists, which provides the upper and the lower estimations of the system's state.

In this paper, the following notation is used. $\mathbb{R}$ denotes the set of real numbers and $\mathbb{R}_{+}=\{x \in \mathbb{R}: x \geq 0\}$. $a \mathscr{R} b$ represents the element-wise relation $\mathscr{R}$ ( $a$ and $b$ are vectors or matrices): for example $a<b$ (vectors) means $\forall i: a_{i}<b_{i}$. For a matrix $\mathscr{A} \in \mathbb{R}^{m \times n}$, define $\mathscr{A}^{+}=\max \{0, \mathscr{A}\}^{1}$ and $\mathscr{A}^{-}=\mathscr{A}^{+}-\mathscr{A}$. For a vector $x \in \mathbb{R}^{n}$, define $x^{+}=\max \{0, x\}$ and $x^{-}=x^{+}-x$. For a matrix (function) $\mathscr{A}$ the symbol $\mathscr{A}_{i}$ denotes its $i^{\text {th }}$ column, for a vector (function) $b$ the symbol $b_{i}$ denotes its corresponding element. A matrix $\mathscr{A} \in \mathbb{R}^{n \times n}$ is called Metzler if all its elements outside the main diagonal are nonnegative. Due to the limitation of pages, the proofs of the results are omitted.

\section{PRoblem STAtement AND BACKGRoundS}

\section{A. Problem statement}

Consider the following uncertain nonlinear singular system:

$$
\Sigma_{\xi}:\left\{\begin{array}{l}
\bar{E} \dot{\xi}=\bar{A} \xi+\bar{f}(\xi, u)+v(t) \\
y=\bar{C} \xi+w(t)
\end{array}\right.
$$

\footnotetext{
${ }^{1}$ It means each element of $\mathscr{A}^{+}$is the maximum value between 0 and the maximum value of $\mathscr{A}$ at the same position.
} 
where $\xi \in \mathbb{R}^{n}$ is the state whose initial value belongs to a compact set $I_{0}\left(\xi\left(t_{0}\right)\right)=\left[\xi\left(t_{0}\right), \bar{\xi}\left(t_{0}\right)\right] ; y \in \mathbb{R}^{p}$ and $u \in \mathbb{R}^{m}$ are respectively the output and the input. $\bar{E} \in \mathbb{R}^{n \times n}, \bar{A} \in \mathbb{R}^{n \times n}$, $\bar{C} \in \mathbb{R}^{p \times n}$, and the vector field $\bar{f}$ represents the nonlinear term with the appropriate dimension. $w(t)$ and $v(t)$ are the disturbance in the output and in the model, respectively.

When the matrix $\bar{E}$ is nonsingular, then (1) can be written as:

$$
\left\{\begin{array}{l}
\dot{\xi}=\bar{E}^{-1} \bar{A} \xi+\bar{E}^{-1} \bar{f}(\xi, u)+\bar{E}^{-1} v(t) \\
y=\bar{C} \xi+w(t)
\end{array}\right.
$$

which becomes a classical regular system. We can cite lots of existing methods to design an observer for the system without the uncertainties, if it is observable. For the case with uncertainties in the state and in the output, an interval observer was proposed in [21] for the observable system (2) to give the upper and lower estimation of the real state with the only requirement of the uncertainty boundedness. This method is extended in [23] to treat the case even if the system is not observable. When the matrix $\bar{E}$ is singular, (1) represents a large class of nonlinear singular systems with uncertainties in the state and in the output. This paper is devoted to designing an interval observer for this larger class of uncertain nonlinear singular systems. Before this, let us firstly recall the basic background on comparison systems which will be used for interval observer design.

\section{ASSUMPTIONS AND PRELIMINARY RESULTS}

Concerning the observability for singular systems, there exist several different definitions in the literature, including observability, R-observability and Impulse-observability [22], [10]. Generally speaking, they characterize the state reconstruction ability from different aspects: R-observability defines the ability to estimate the reachable set of the studied system; Impulse-observability corresponds to the ability to estimate the impulse term of the studied system and the observability covers both mentioned abilities to estimate all states of the studied system.

In this paper, for the uncertain nonlinear singular system $\Sigma_{\xi}$, we are interested in the interval estimation of the (non-impulsive) trajectory of the state $\xi(t)$ with the known information of $y(t)$ and $u(t)$ for $t>0$. Therefore, this paper considers the following definition of observability adopted from Definition 1 in [1].

Definition 1: System $\Sigma_{\xi}$ is observable if $y\left(\xi_{1}, t\right)=y\left(\xi_{2}, t\right)$ for all $\xi_{1}, \xi_{2} \in \mathbb{R}^{n}$ and $t>0$ implies $\xi_{1}\left(0^{+}\right)=\xi_{2}\left(0^{+}\right)$.

In what follows, we make the following assumption for the original system $\Sigma_{\xi}$.

Assumption 1: For the triple $(\bar{E}, \bar{A}, \bar{C})$ of system $\Sigma_{\xi}$ defined in (1), it is assumed that the following rank conditions:

$$
\operatorname{rank}\left[\begin{array}{c}
\bar{E} \\
\bar{C}
\end{array}\right]=n
$$

and

$$
\operatorname{rank}\left[\begin{array}{c}
s \bar{E}-\bar{A} \\
\bar{C}
\end{array}\right]=n, \forall s \in \mathbb{C}
$$

are satisfied.
It is obvious that, even if the rank conditions (3) and (4) are assumed to be satisfied, the studied uncertain nonlinear singular system $\sum_{\xi}$ might not be observable, due to the nonlinear term $\bar{f}(\xi, u)$ and the uncertainties $(v, w)$. And this motivates the study of the interval estimation treated in this paper. Without loss of generalities, for the singular matrix $\bar{E} \in \mathbb{R}^{n \times n}$, it is assumed that $\operatorname{rank} \bar{E}=q<n$. Due to the rank condition (3), there exists a non-singular matrix $P=\left[\begin{array}{ll}P_{1} & P_{2} \\ P_{3} & P_{4}\end{array}\right] \in \mathbb{R}^{(p+n) \times(p+n)}$ such that $P\left[\begin{array}{c}\bar{E} \\ \bar{C}\end{array}\right]=\left[\begin{array}{c}I_{n} \\ 0\end{array}\right]$, which is equivalent to:

$$
\left\{\begin{array}{c}
P_{1} \bar{E}+P_{2} \bar{C}=I_{n} \\
P_{3} \bar{E}+P_{4} \bar{C}=0
\end{array}\right.
$$

with $P_{1} \in \mathbb{R}^{n \times n}, P_{2} \in \mathbb{R}^{n \times p}, P_{3} \in \mathbb{R}^{p \times n}$ and $P_{4} \in \mathbb{R}^{p \times p}$. Based on the above result, we have the following lemma.

Lemma 1: Suppose Assumption 1 is satisfied for the triple $(\bar{E}, \bar{A}, \bar{C})$ of $\Sigma_{\xi}$. Then there always exist matrices $K \in \mathbb{R}^{n \times p}$, $L \in \mathbb{R}^{n \times p}$ and an invertible matrix $Q \in \mathbb{R}^{n \times n}$ such that the following matrix:

$$
R=Q N Q^{-1}
$$

is Hurwitz and Metzler, where

$$
N=\left(P_{1}+K P_{3}\right) \bar{A}+L \bar{C},
$$

with $P_{1}$ and $P_{3}$ being defined in (5).

With the above deduced matrix $Q$ which transforms the Hurwitz matrix $N$ to the Hurwitz and Metzler matrix $R$, since it is non-singular, then we can choose $x=Q \xi$ as a diffeomorphism, with which system $\Sigma_{\xi}$ in (1) can be rewritten as follows:

$$
\Sigma_{x}:\left\{\begin{array}{l}
E \dot{x}=A x+f(x, u)+v(t) \\
y=C x+w(t)
\end{array}\right.
$$

where $E=\bar{E} Q^{-1}, A=\bar{A} Q^{-1}, C=\bar{C} Q^{-1}$ and $f(x, u)=$ $\bar{f}\left(Q^{-1} x, u\right)$. For the transformed system (8), we can state the following lemma.

Lemma 2: Suppose Assumption 1 is satisfied for the triple $(\bar{E}, \bar{A}, \bar{C})$ of $\Sigma_{\xi}$. Then for the transformed system $\Sigma_{x}(E, A, C)$ defined in (8), there always exist a matrix $K \in \mathbb{R}^{n \times p}$, two invertible matrices $P=\left[\begin{array}{ll}P_{1} & P_{2} \\ P_{3} & P_{4}\end{array}\right] \in \mathbb{R}^{(p+n \times(p+n)}$ and $Q \in$ $\mathbb{R}^{n \times n}$, such that the following equality is satisfied:

$$
Q P_{24} C+Q P_{13} E=I_{n}
$$

where the matrices $P_{13}$ and $P_{24}$ are defined as follows:

$$
\begin{aligned}
& P_{13}=P_{1}+K P_{3} \\
& P_{24}=P_{2}+K P_{4}
\end{aligned}
$$

Since the initial condition $\xi_{0}$ for (1) is supposed to be located into a certain interval $I\left(\xi_{0}\right)=\left[\underline{\xi_{0}}, \overline{\xi_{0}}\right]$, then using the diffeomorphism $x=Q \xi$, the initial condition $x_{0}$ is also known within a certain interval $I\left(x_{0}\right)=\left[\underline{x}_{0}, \bar{x}_{0}\right]$. Thus the interval estimation of $\xi$ in (1) is equivalent to estimate the interval of $x$ in (8) by using the knowledge of $(u, y)$. 


\section{MAIN RESUlts}

This section will firstly analyze the boundedness properties by making some assumptions for (1), then propose an interval observer to estimate the state.

\section{A. Interval estimation}

Prior to introduce the interval observer for (1), let us firstly make the following assumption which is necessary in the sequel.

Assumption 2: For the studied system (1), it is assumed that:

1) the state $\xi(t)$ is bounded under the bounded input $u(t) \in$ $C^{\infty}$, i.e. $\xi(t) \in \Omega \subset \mathbb{R}^{n}$ for a given $u(t) \in \mathscr{U} \subset \mathbb{R}^{m}$ for all $t \geq 0$ where $\Omega$ and $\mathscr{U}$ are two given compact sets;

2) the function $\bar{f}(\xi, u)$ for all $\xi \in \Omega$ and $u \in \mathscr{U}$ is locally Lipschtiz w.r.t $(\xi, u)$;

3) the disturbances $w(t)$ and $v(t)$ are bounded, and the derivative of $w(t)$ is bounded for all $t \geq 0$, i.e. there exist constants $\bar{v}, \underline{v}, \bar{w}, \underline{w}, \overline{w_{d}}, \underline{w_{d}}$ such that $\underline{v} \leq v(t) \leq \bar{v}$, $\underline{w} \leq w(t) \leq \bar{w}$, and $w_{d} \leq \dot{w}(t) \leq \overline{w_{d}}$.

Remark 1: Since system (8) was transformed from the system (1) by applying the diffeomorphism $x=Q \xi: \Omega \rightarrow \mathscr{X}$, therefore if Assumption 2 is fulfilled for (1), then the state $x(t)$ of (8) is bounded as well for the bounded input $u(t)$, i.e. $x(t) \in \mathscr{X} \subset \mathbb{R}^{n}$ for all $t \geq 0$ where $\mathscr{X}$ represents the compact set defined via the diffeomorphism. Moreover, since $f(x, u)=\bar{f}\left(Q^{-1} x, u\right)$ in (8), thus it is locally Lipschtiz w.r.t $(x, u)$ for all $x \in \mathscr{X}$ and $u \in \mathscr{U}$.

As we have showed in Section III, if Assumption 1 and Assumption 2 are satisfied, then there exist the matrices $K, L$, and the non-singular matrices $P$ and $Q$ such that the matrix $N$ defined in (7) is Hurwitz, and the matrix $R$ defined in (6) is Hurwitz and Metzler. For the sake of simplicity, note

$$
\begin{aligned}
& Q_{P_{13}}=Q P_{13} \\
& Q_{P_{24}}=Q P_{24} \\
& Q_{L}=Q L
\end{aligned}
$$

where $P_{13}$ and $P_{24}$ are defined in (9). Then one can design the following two dynamics:

$$
\left\{\begin{array}{l}
\dot{\bar{z}}=R \bar{z}+\left(R Q_{P_{24}}-Q_{L}\right) y+\bar{\Delta}(\underline{z}, \bar{z}, u) \\
\bar{x}=\bar{z}+Q_{P_{24}} y
\end{array}\right.
$$

and

$$
\left\{\begin{array}{l}
\dot{\dot{z}}=R_{\underline{z}}+\left(R Q_{P_{24}}-Q_{L}\right) y+\underline{\Delta}(\underline{z}, \bar{z}, u) \\
\underline{x}=\underline{z}+Q_{P_{24}} y
\end{array}\right.
$$

with

$$
\begin{aligned}
& \bar{\Delta}(\underline{z}, \bar{z}, u)=\overline{\Delta_{f}}(\underline{z}, \bar{z}, u)+\overline{\Delta_{v}}+\overline{\Delta_{w}}+\overline{\Delta_{w_{d}}} \\
& \overline{\Delta_{f}}(\underline{z}, \bar{z}, u)=Q_{P_{13}}^{+} \bar{f}(\underline{z}, \bar{z}, u)-Q_{P_{13}}^{-} \underline{f}(\underline{z}, \bar{z}, u) \\
& \overline{\Delta_{v}}=Q_{P_{13}}^{+} \bar{v}-Q_{P_{13}}^{-} \underline{v} \\
& \overline{\Delta_{w}}=Q_{L}^{+} \bar{w}-Q_{L}^{-} \underline{w} \\
& \overline{\Delta_{w_{d}}}=Q_{P_{24}}^{-} \overline{w_{d}}-Q_{P_{24}}^{+} \frac{w_{d}}{}
\end{aligned}
$$

and

$$
\begin{aligned}
& \underline{\Delta}(\underline{z}, \bar{z}, u)=\underline{\Delta_{f}}(\underline{z}, \bar{z}, u)+\underline{\Delta_{v}}+\underline{\Delta_{w}}+\underline{\Delta_{w_{d}}} \\
& \underline{\Delta_{f}}(\underline{z}, \bar{z}, u)=Q_{P_{13}}^{+} \underline{f}(\underline{z}, \bar{z}, u)-Q_{P_{13}}^{-} \bar{f} \frac{(\underline{z}, \bar{z}, u)}{\overline{\Delta_{v}}}=Q_{L}^{+} \underline{v}-Q_{L}^{-} \bar{v} \\
& \underline{\Delta_{w}}=Q_{L}^{+} \underline{w}-Q_{L}^{-} \bar{w} \\
& \underline{\Delta_{w_{d}}}=Q_{P_{24}}^{-} \underline{w_{d}}-Q_{P_{24}}^{+} \overline{w_{d}}
\end{aligned}
$$

\section{By noting}

$$
\begin{aligned}
\bar{\Gamma}(\underline{x}, \bar{x}, x, u)= & \bar{\Delta}(\underline{z}, \bar{z}, u)-Q_{P_{13}} f(x, u)-Q_{P_{13}} v(t) \\
& -Q_{L} w(t)+Q_{P_{24}} \dot{w}(t)
\end{aligned}
$$

and

$$
\begin{aligned}
\underline{\Gamma}(\underline{x}, \bar{x}, x, u)= & -\underline{\Delta}(\underline{z}, \bar{z}, u)+Q_{P_{13}} f(x, u)+Q_{P_{13}} v(t) \\
& +Q_{L} w(x, u)-Q_{P_{24}} \dot{w}(t)
\end{aligned}
$$

we have the following corollary.

Corollary 1: For $\bar{\Gamma}(\underline{x}, \bar{x}, x, u)$ and $\underline{\Gamma}(\underline{x}, \bar{x}, x, u)$ defined in (12) and (13) with $x \in \mathscr{X}$ and $u \in \mathscr{U}$, we have

$$
\bar{\Gamma}(\underline{x}, \bar{x}, x, u) \geq 0 \text { and } \underline{\Gamma}(\underline{x}, \bar{x}, x, u) \geq 0
$$

Moreover, there exist positive constants $\underline{l}_{\bar{\Gamma}}, \bar{l}_{\underline{\Gamma}}, \underline{l}_{\bar{\Gamma}}, \bar{l}_{\bar{\Gamma}}$ and $l_{\bar{\Gamma}}$, $l_{\underline{\Gamma}}$ such that for a chosen submultiplicative norm $\|\cdot\|$ the following inequalities:

$$
\begin{aligned}
&\|\bar{\Gamma}(\underline{x}, \bar{x}, x, u)\| \leq \bar{l}_{\bar{\Gamma}}\|\bar{x}-x\|+\underline{l_{\bar{\Gamma}}}\|x-\underline{x}\|+l_{\bar{\Gamma}} \\
&\|\underline{\Gamma}(\underline{x}, \bar{x}, x, u)\| \leq \bar{l}_{\underline{\Gamma}}\|\bar{x}-x\|+\underline{l}_{\underline{\Gamma}}\|x-\underline{x}\|+l_{\underline{\Gamma}}
\end{aligned}
$$

are satisfied for all $t \geq 0$.

Theorem 1: Suppose Assumption 1 and Assumption 2 are satisfied. Then for any initial state $x\left(t_{0}\right)$ of (8) belongs to a certain interval $I\left(x\left(t_{0}\right)\right)=\left[\underline{x}\left(t_{0}\right), \bar{x}\left(t_{0}\right)\right]$, systems (10) and (11) form an interval observer for (8) such that the following inequality:

$$
\underline{x}(t) \leq x(t) \leq \bar{x}(t),
$$

holds for all $t \geq t_{0}$. Mover, if there exist positive definite symmetric matrices $S, M$ and a positive scalar $\mu$ such that the following LMI is satisfied:

$$
\left[\begin{array}{cc}
\mathscr{R}^{T} S+S \mathscr{R}+\frac{\alpha}{\mu} I+M & S \\
S & -\frac{1}{\mu} I
\end{array}\right] \preceq 0
$$

where $\mathscr{R}=\operatorname{diag}\{R, R\}, \alpha=2 \max \left\{\underline{l}_{\underline{\Gamma}}^{2}, \bar{l}_{\bar{\Gamma}}^{2}, \underline{l}_{\bar{\Gamma}}^{2}, \bar{l}_{\bar{\Gamma}}^{2}, l_{\bar{\Gamma}}^{2}, l_{\underline{\Gamma}}^{2}\right\}$, then the variables $\underline{x}(t)$ and $\bar{x}(t)$ are bounded for all $t \geq 0$.

Corollary 2: For system (8) with the initial state $\xi\left(t_{0}\right) \in$ $\left[\xi\left(t_{0}\right), \bar{\xi}\left(t_{0}\right)\right]$, if Assumption 1 and Assumption 2 are satisfied, then there exists a non-singular matrix $Q$ such that the interval estimation of $\xi$ in (1) is given as follows:

$$
\left[Q^{-1}\right]^{+} \underline{x}-\left[Q^{-1}\right]^{-} \bar{x} \leq \xi \leq\left[Q^{-1}\right]^{+} \bar{x}-\left[Q^{-1}\right]^{-} \underline{x}
$$

where $\underline{x}$ and $\bar{x}$ are the states of the proposed interval observer defined in (10) and (11).

Let us remark that Assumption 2 imposed the state boundedness $\xi(t) \in \Omega \subset \mathbb{R}^{n}$ under a given bounded input $u(t) \in \mathscr{U} \subset \mathbb{R}^{m}$ for all $t \geq 0$, and this property is sometimes difficult to be checked for general uncertain nonlinear singular systems, therefore the proposed result could not be applied. However, if the boundedness property of the state in Assumption 2 is satisfied only for $t \in[0, T]$ with $T$ being a finite time, i.e. $\xi(t) \in \Omega \subset \mathbb{R}^{n}$ under a given bounded input $u(t) \in \mathscr{U} \subset \mathbb{R}^{m}$ for all $t \in[0, T]$, then Theorem 1 is still valid during this finite time $T$. Therefore the proposed result can be relaxed by the following corollary.

Corollary 3: Suppose Assumption 1 and Assumption 2 are valid only for $t \in[0, T]$ with $T$ being a finite time. Then for any initial state $x\left(t_{0}\right)$ of (8) belongs to a certain interval 
$I\left(x\left(t_{0}\right)\right)=\left[\underline{x}\left(t_{0}\right), \bar{x}\left(t_{0}\right)\right]$, systems (10) and (11) give an interval estimation of $x(t)$ of (8) for $t \in\left[t_{0}, T\right]$, i.e. $\underline{x}(t) \leq x(t) \leq \bar{x}(t)$ for all $t \in\left[t_{0}, T\right]$. And the interval estimation of $\xi(t)$ of (1) for all $t \in\left[t_{0}, T\right]$ can be still obtained by (16).

\section{B. Design procedure}

For system $\sum_{\xi}(\bar{E}, \bar{A}, \bar{C})$ defined in (1), supposed that Assumption 1 and Assumption 2 are both satisfied for all $t \geq 0$ (or for all $t \in[0, T]$ ), then the interval estimation of the state $\xi$ for (1) can be obtained via the following procedure:

Step1: Due to the satisfaction of the rank condition (3) in Assumption 1, determine an invertible matrix $P=$ $\left[\begin{array}{ll}P_{1} & P_{2} \\ P_{3} & P_{4}\end{array}\right]$ such that $P\left[\begin{array}{c}\bar{E} \\ \bar{C}\end{array}\right]=\left[\begin{array}{c}I_{n} \\ 0\end{array}\right]$

Step2: Due to the satisfaction of the rank condition (4) in Assumption 1, choose the matrices $K, L$ such that $N=$ $L \bar{C}+\left(P_{1}+K P_{3}\right) \bar{A}$ is Hurwitz;

Step3: Choose an invertible matrix $Q$ such that $R=Q N Q^{-1}$ is Hurwitz and Metzler;

Step4: Transform system $\bar{\Sigma}_{\xi}(\bar{E}, \bar{A}, \bar{C})$ into $\Sigma_{x}(E, A, C)$ by applying the diffeomorphism $x=Q \xi$;

Step5: If Assumption 2 is satisfied for all $t \geq 0$ (or for all $t \in[0, T]$, calculate the bound of the function $f$ in (8);

Step6: Design interval observer (10) and (11) for $\Sigma_{x}(E, A, C)$, which yields $\underline{x}(t) \leq x(t) \leq \bar{x}(t)$ for all $t \geq 0$ (or for all $t \in[0, T])$;

Step7: Finally we obtain the interval estimation of $\xi(t)$ for all $t \geq 0$ (or for all $t \in[0, T]$ ):

$$
\left[Q^{-1}\right]^{+} \underline{x}-\left[Q^{-1}\right]^{-} \bar{x} \leq \xi \leq\left[Q^{-1}\right]^{+} \bar{x}-\left[Q^{-1}\right]^{-} \underline{x} .
$$

\section{ILLUSTRATIVE EXAMPLE}

Let us consider the following uncertain nonlinear singular system:

$$
\begin{aligned}
& \bar{E} \dot{\xi}=\bar{A} \xi+\bar{f}(\xi, u)+v(t) \\
& y=\bar{C} \xi+w(t)
\end{aligned}
$$

with

$$
\begin{aligned}
& \bar{E}=\left[\begin{array}{lll}
0 & 1 & 0 \\
0 & 0 & 1 \\
0 & 0 & 0
\end{array}\right], \bar{A}=\left[\begin{array}{ccc}
20 & 0 & 0 \\
1 & 8 & 0 \\
1 & 0 & 1
\end{array}\right] \text { and } \bar{C}=\left[\begin{array}{l}
1 \\
0 \\
0
\end{array}\right]^{T} \\
& \bar{f}(\xi, u)=\left[\begin{array}{c}
\sin \left(\frac{\xi_{3}}{32}\right) \\
u \sin \left(\frac{4 \xi_{2}-\xi_{3}}{240}\right) \\
-0.75 \sin \left(\frac{4 \xi_{2} \xi_{3}-\xi_{3}^{2}}{120}\right)
\end{array}\right] \quad \text { and } v(t)= \\
& {\left[\begin{array}{c}
0.1 \cos 3 t \\
0.2 \sin 4 t \\
0.15 \sin 2 t
\end{array}\right], \quad w(t)=0.1 \sin (t) \text {. It is straightforward }}
\end{aligned}
$$

to check that the studied uncertain nonlinear singular system may b not observable with respect to the output $y$, thus an asymptotic observer could not be designed. The following applies the proposed method to design an interval observer to get the upper and the lower estimations of the state.

Step 1:

Let us firstly check the rank conditions imposed in Assumption 1. Since

$$
\operatorname{rank}\left[\begin{array}{c}
\bar{E} \\
\bar{C}
\end{array}\right]=3
$$

and

$$
\operatorname{rank}\left[\begin{array}{c}
s \bar{E}-\bar{A} \\
\bar{C}
\end{array}\right]=3, \forall s \in \mathbb{C}
$$

thus Assumption 1 is fulfilled. Moreover, the input is chosen as $u(t)=0.5 \sin (t)$ such that Assumption 2 is at least satisfied for $t \in[0, T]$ with $T$ being a finite time (see Fig. 1-3 for $T=5)$.

Then one can find an invertible matrix $P=$ $\left[\begin{array}{llll}0 & 0 & 0 & 1 \\ 1 & 0 & 0 & 0 \\ 0 & 1 & 0 & 0 \\ 0 & 0 & 1 & 0\end{array}\right]=\left[\begin{array}{ll}P_{1} & P_{2} \\ P_{3} & P_{4}\end{array}\right]$ with $P_{1}=\left[\begin{array}{lll}0 & 0 & 0 \\ 1 & 0 & 0 \\ 0 & 1 & 0\end{array}\right]$ $P_{2}=\left[\begin{array}{l}1 \\ 0 \\ 0\end{array}\right], P_{3}=\left[\begin{array}{lll}0 & 0 & 1\end{array}\right]$ and $P_{4}=\left[\begin{array}{l}0\end{array}\right]$ such that $P\left[\begin{array}{c}\bar{E} \\ \bar{C}\end{array}\right]=\left[\begin{array}{c}I_{3 \times 3} \\ 0\end{array}\right]$.

\section{Step 2:}

Therefore, we can choose:

$$
K=[0,-1,-6]^{T}, \quad L=[-2,-19,5]^{T}
$$

such that $N=L \bar{C}+\left(P_{1}+K P_{3}\right) \bar{A}=\left[\begin{array}{ccc}-2 & 0 & 0 \\ 0 & 0 & -1 \\ 0 & 8 & -6\end{array}\right]$ with negative eigenvalues $(-2,-4,-2)$.

\section{Step 3:}

Hence, we can find the following invertible matrix:

$$
Q=\left[\begin{array}{ccc}
1 & 0 & 0 \\
0 & 1 & -0.25 \\
0 & 0 & 1
\end{array}\right]
$$

such that $R=Q N Q^{-1}=\left[\begin{array}{ccc}-2 & 0 & 0 \\ 0 & -2 & 0 \\ 0 & 8 & -4\end{array}\right]$ which is Hurwitz and Metzler.

\section{Step 4:}

So, with the transformation $x=Q \xi$, system (17) can be written as:

$$
\begin{aligned}
& E \dot{x}=A x+f(x, u)+v(t) \\
& y=C x+w(t)
\end{aligned}
$$

with $E=\bar{E} Q^{-1}=\left[\begin{array}{ccc}0 & 1 & 0.25 \\ 0 & 0 & 1 \\ 0 & 0 & 0\end{array}\right], \quad A=\bar{A} Q^{-1}=$ $\left[\begin{array}{ccc}20 & 0 & 0 \\ 1 & 8 & 2 \\ 1 & 0 & 1\end{array}\right], \quad C=\bar{C} Q^{-1}=\left[\begin{array}{lll}1 & 0 & 0\end{array}\right] \quad$ and $f(x, u)=\bar{f}\left(Q^{-1} x, u\right)=\left[\begin{array}{c}\sin \left(\frac{x_{3}}{32}\right) \\ u \sin \left(\frac{x_{2}}{60}\right) \\ -0.75 \sin \left(\frac{x_{2} x_{3}}{30}\right)\end{array}\right]$.

\section{Step 5:}

Let us now compute the bounding function for $f(x, u)$ in (18). To this end, define the following two functions:

$$
\begin{aligned}
\operatorname{Product}(\underline{x}, \bar{x}) & =\left[\begin{array}{l}
\min \left\{\bar{x}_{2} \bar{x}_{3}, \underline{x}_{2} \bar{x}_{3}, \bar{x}_{2} \underline{x}_{3}, \underline{x}_{2} \underline{x}_{3}\right\} \\
\max \left\{\bar{x}_{2} \bar{x}_{3}, \underline{x}_{2} \bar{x}_{3}, \bar{x}_{2} \underline{x}_{3}, \underline{x}_{2} \underline{x}_{3}\right\}
\end{array}\right], \\
{\left[\begin{array}{c}
\underline{\sin }(\underline{x}, \bar{x}) \\
\overline{\sin }(\underline{x}, \bar{x})
\end{array}\right] } & =\left[\begin{array}{c}
\sin (\underline{x}) \\
\sin (\bar{x})
\end{array}\right]
\end{aligned}
$$


corresponding to the interval of the product $x_{2} x_{3}$ for $x=$ $\left[\begin{array}{ll}x_{2} & x_{3}\end{array}\right]^{T}$ with $\underline{x} \leq x \leq \bar{x}$ and the interval of the function $\sin (x)$ for a scalar $x$ with $\underline{x} \leq x \leq \bar{x}$ (for $|x| \leq \pi / 2$ ). Then we have the following bounding functions for $f$ :

$$
\begin{aligned}
& \underline{f}_{1}(\underline{x}, \bar{x}) \quad=\underline{\sin }(\underline{x} / 32, \bar{x} / 32) \\
& \overline{\bar{f}}_{1}(\underline{x}, \bar{x}) \quad=\overline{\overline{\sin }}(\underline{x} / 32, \bar{x} / 32) \\
& {\left[\begin{array}{c}
f_{2}(\underline{x}, \bar{x}) \\
\bar{f}_{2}(\underline{x}, \bar{x})
\end{array}\right]=\operatorname{Product}\left(\left[\begin{array}{c}
\underline{u} \\
\bar{u}
\end{array}\right],\left[\begin{array}{l}
\overline{\sin }(\underline{x} / 60, \bar{x} / 60) \\
\overline{\sin }(\underline{x} / 60, \bar{x} / 60)
\end{array}\right]\right)} \\
& \underline{f}_{3}(\underline{x}, \bar{x}) \quad=-0.75 \overline{\sin }(\operatorname{Product}(\underline{x} / 30, \bar{x} / 30)) \\
& \overline{\bar{f}}_{3}(\underline{x}, \bar{x}) \quad=-0.75 \underline{\sin }(\operatorname{Product}(\underline{x} / 30, \bar{x} / 30))
\end{aligned}
$$

Take $\underline{l}_{\underline{\Gamma}}=\bar{l}_{\underline{\Gamma}}=\underline{l}_{\bar{\Gamma}}=\bar{l}_{\bar{\Gamma}}=l_{\bar{\Gamma}}=l_{\underline{\Gamma}}=1$, then we have $\alpha=2$. By solving the LMI defined in (15), we find $\mu=1, S=$ $\operatorname{diag}\left\{\mathrm{S}_{1}, \mathrm{~S}_{1}\right\}, M=\operatorname{diag}\left\{\mathrm{M}_{1}, \mathrm{M}_{1}\right\}$ with

$$
S_{1}=\left[\begin{array}{ccc}
1.6 & 0 & 0 \\
0 & 3 & 0.4 \\
0 & 0.4 & 0.5
\end{array}\right], M_{1}=\left[\begin{array}{ccc}
3.5 & 0 & 0 \\
0 & 4.3 & 0.2 \\
0 & 0.2 & 3.4
\end{array}\right],
$$

thus all conditions of Theorem 1 have been verified.

\section{Step 6:}

Then we can design the interval observer for the transformed system (18). The results of the interval estimations of the state $x$ for this system are depicted in Fig. 1-3, from which we can see that the proposed observer can provide both the upper and the lower estimations of $x$.

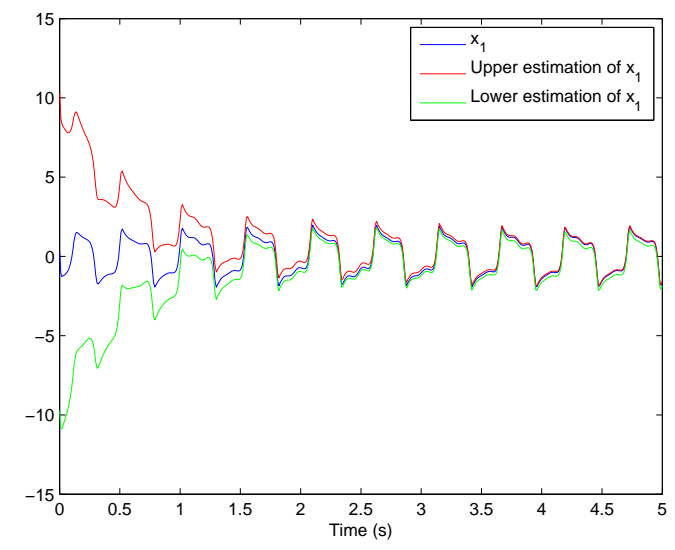

Fig. 1. The interval estimation for $x_{1}$.

\section{Step 7:}

Then, by applying

$$
\left[Q^{-1}\right]^{+} \underline{x}-\left[Q^{-1}\right]^{-} \bar{x} \leq \xi \leq\left[Q^{-1}\right]^{+} \bar{x}-\left[Q^{-1}\right]^{-} \underline{x}
$$

we obtain the upper and the lower estimation of the real state $\xi$ for the uncertain nonlinear singular systems (17) (see Fig. 4-6).

\section{CONCLUSION}

When treating the uncertain nonlinear singular system while the asymptotic estimation is not possible, this paper is devoted to designing an interval observer for the studied uncertain system. By imposing the rank conditions and assuming the boundedness of the uncertainties (and

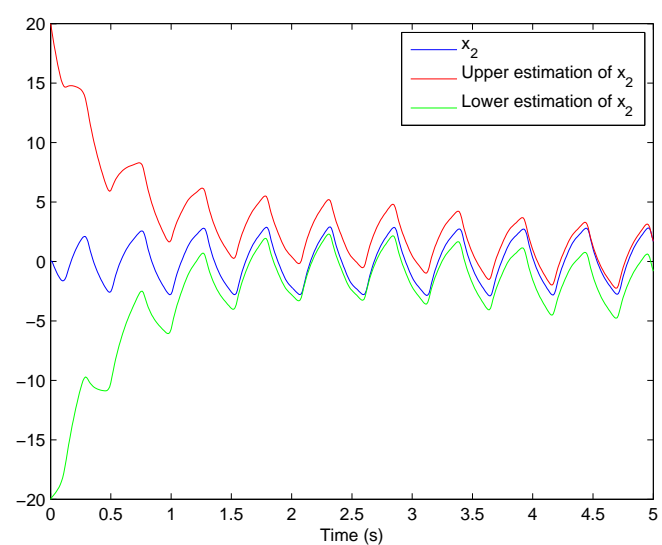

Fig. 2. The interval estimation for $x_{2}$.

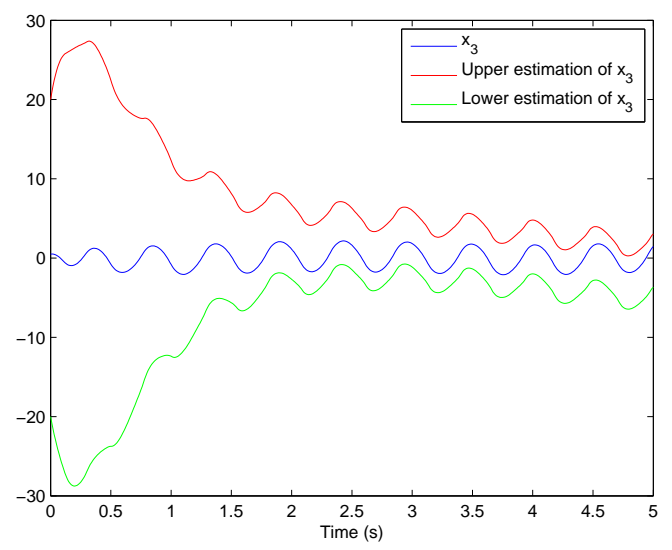

Fig. 3. The interval estimation for $x_{3}$.

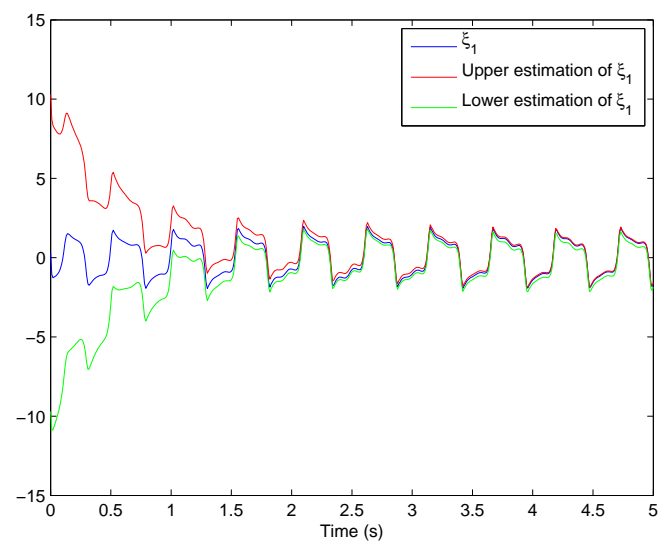

Fig. 4. The interval estimation for $\xi_{1}$.

the derivative of the uncertainty) in the state and in the output, we proved that an interval observer can always be synthesized to provide the upper and the lower estimations of the real state. The efficiency of the proposed method is highlighted by an illustrative example. 


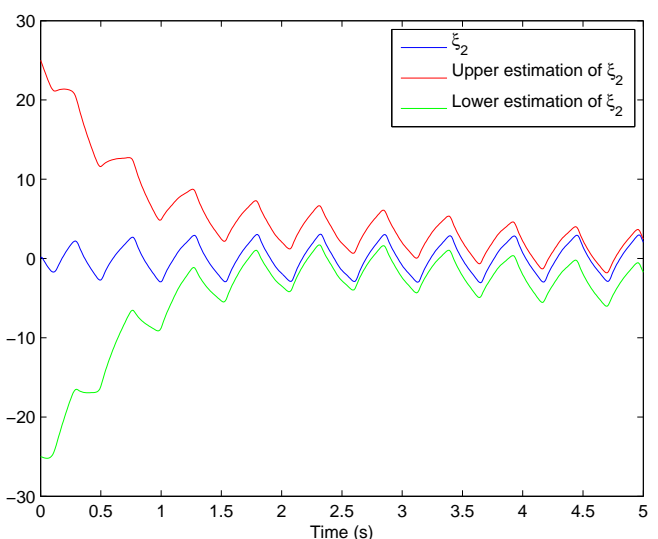

Fig. 5. The interval estimation for $\xi_{2}$.

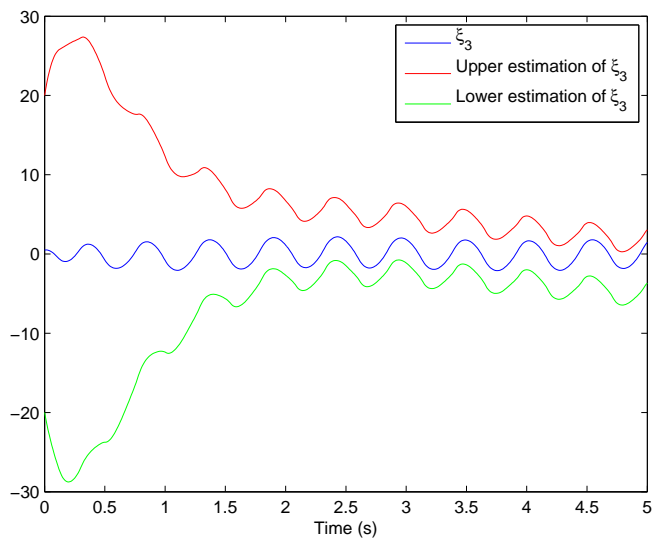

Fig. 6. The interval estimation for $\xi_{3}$.

\section{REFERENCES}

[1] F. J. Bejarano, Thierry Floquet, Wilfrid Perruquetti, and Gang Zheng. Observability and detectability of singular linear systems with unknown inputs. Automatica, 49(3):793-800, 2013.

[2] F. J. Bejarano, Thierry Floquet, Wilfrid Perruquetti, and Gang Zheng. Observation of nonlinear differential-algebraic systems with unknown inputs. IEEE Transactions on Automatic Control, 60(7):1957-1962, 2015.

[3] F.J. Bejarano, T. Floquet, W. Perruquetti, and Gang Zheng. Observability and detectability analysis of singular linear systems with unknown inputs. In Decision and Control and European Control Conference (CDC-ECC), 2011 50th IEEE Conference on, pages 4005-4010, 2011.

[4] F.J. Bejarano, W. Perruquetti, T. Floquet, and Gang Zheng. State reconstruction of nonlinear differential-algebraic systems with unknown inputs. In Decision and Control (CDC), 2012 IEEE 51st Annual Conference on, pages 5882-5887, 2012.

[5] Driss Boutat, Gang Zheng, Latifa Boutat-Baddas, and Mohamed Darouach. Observers design for a class of nonlinear singular systems. In in Proc. IEEE Conf. Decision Control, 2012.

[6] M. Boutayeb and M. Darouach. Observers design for non linear descriptor systems. In in Proc. IEEE Conf. Decision Control, 1995.

[7] S.L. Campbell. Singular systems of differential equations I. Pitman, 1980.

[8] S.L. Campbell. Singular systems of differential equations II. Pitman, 1982.

[9] D. Cobb. Controllability, observability, and duality in singular systems. IEEE Transactions on Automatic Control, AC-29(12):10761082, 1984.
[10] L. Dai. Singular Control Systems, volume 118. Lecture Notes in Control and Information Sciences, Springer-Verlag, New York, 1989.

[11] M. Darouach and M. Boutayeb. Design of observers for descriptor systems. IEEE Trans. Automatic Control, 40(7):1323-1327, 1995.

[12] M. Darouach, M. Zasadzinski, and M. Hayar. Reduced-order observer design for descriptor systems with unknown inputs. IEEE Transactions on Automatic Control, 41(7):1068-1072, 1996.

[13] D. Efimov, A. Polyakov, and J.-P. Richard. Interval estimation for systems with time delays and algebraic constraints. In in Proc. of ECC, 2014.

[14] J.-L. Gouzé, A. Rapaport, and Z. Hadj-Sadok. Interval observers for uncertain biological systems. Ecological Model, 133:45-56, 2000.

[15] M. Hou and P. C. Muller. Casual observability of descriptor systems. IEEE Transactions on Automatic Control, 44(1):158-163, 1999.

[16] M. Hou and P. C. Muller. Observer design for descriptor systems. IEEE Transactions on Automatic Control, 44(1):164-169, 1999.

[17] S. Kaprielian and J. Turi. An observer for a nonlinear descriptor system. in Proc. IEEE Conf. Decision Control, 1992.

[18] P.N. Paraskevopoulos and F.N. Koumboulis. Observers for singular systems. IEEE Transactions on Automatic Control, 37(8):1211 - 1215, August 1992.

[19] W Perruquetti and JP Richard. Connecting wasewskis conditions with mmatrices: Application to constrained stabilization. Dynam. Syst. Appl, 5:81-96, 1996.

[20] W Perruquetti, JP Richard, Lj T Grujić, and P Borne. On practical stability with the settling time via vector norms. International Journal of Control, 62(1):173-189, 1995.

[21] T. Raïssi, D. Efimov, and A. Zolghadri. Interval state estimation for a class of nonlinear systems. IEEE Trans. Automatic Control, 57:260265, 2012.

[22] E. Yip and R.F. Sincovec. Solvability, controllability, and observability of continuous descriptor systems. IEEE Transactions on Automatic Control, AC-26(3):702-707, 1981.

[23] Gang Zheng, Denis Efimov, and Wilfrid Perruquetti. Interval state estimation for uncertain nonlinear systems. In IFAC, NOLCOS, 2013. 